\title{
ESTUDO DA QUALIDADE DE VIDA EM INDIVÍDUOS COM PARALISIA FACIAL PERIFÉRICA CRÔNICA ADQUIRIDA
}

\author{
Study on quality of life in subjects with acquired \\ chronic peripheral facial palsy
}

Rayné Moreira Melo Santos ${ }^{(1)}$, Zelita Caldeira Ferreira Guedes ${ }^{(2)}$

\begin{abstract}
RESUMO
Objetivo: analisar a qualidade de vida em indivíduos com paralisia facial periférica crônica adquirida. Método: foram selecionados, por meio de triagem, 12 indivíduos com paralisia facial periférica adquirida na fase de sequela, com etiologias de paralisia facial de Bell e Schwannoma após exérese. Foi verificado o grau da paralisia facial periférica adquirida de acordo com House \& Brackmann ${ }^{9}$, além de ter sido realizada entrevista com perguntas fechadas, para verificar se havia interferência da paralisia facial na convivência social de cada indivíduo. O tipo de estudo foi transversal e, os testes utilizados foram o não-paramétrico de Mann-Whitney e o teste exato de Fisher, com o nível de significância de 5\%. Resultados: os graus da paralisia facial foram divididos da seguinte forma: I-II (Normal a Disfunção leve), III-IV (Disfunção moderada a moderadamente severa) e V-VI (Disfunção severa a paralisia total). Nas respostas quanto ao prejuízo nas atividades profissionais e pessoais, indivíduos com face normal a disfunção leve por paralisia facial de Bell responderam não ter prejuízo para suas atividades; na disfunção moderada a moderadamente severa todos responderam muito prejuízo e na disfunção severa a paralisia total, um indivíduo respondeu muito prejuízo. Na paralisia facial por Schwannoma no grupo classificado como disfunção leve, todos responderam nenhum prejuízo e na disfunção severa à paralisia total, um indivíduo respondeu muito prejuízo para tais atividades. Conclusão: a paralisia facial periférica crônica adquirida interferiu na qualidade de vida dos indivíduos com graus considerados mais graves.
\end{abstract}

DESCRITORES: Qualidade de Vida; Paralisia Facial; Paralisia de Bell; Expressão Facial; Impacto Psicossocial

\section{INTRODUÇÃO}

A preocupação com a satisfação do desempenho funcional e a qualidade de vida (QV) tem crescido nos últimos anos. A extensão desses comprometimentos é reconhecida pela American

(1) Fonoaudióloga; Coordenadora do Serviço de Fonoaudiologia do Centro de Reabilitação da Associação dos Deficientes Físicos de Alagoas, CREMGEC/ADEFAL, Maceió, AL, Brasil; Fonoaudióloga da Secretaria de Estado da Educação do Estado de Alagoas, SEE, Maceió, AL, Brasil; Mestranda em Distúrbios da Comunicação Humana pela Universidade Federal de São Paulo - UNIFESP.

(2) Fonoaudióloga; Professora Doutora Associada do Departamento de Fonoaudiologia da Universidade Federal de São Paulo - UNIFESP, São Paulo, SP, Brasil; Pós-Doutorado na Universidade René Descartes, Paris 5, França.

Conflito de interesses: inexistente
Medical Association Guides to the Evaluation of Permanent Impairment ${ }^{1,2}$.

A qualidade de vida reflete a percepção de que as necessidades dos indivíduos estão sendo satisfeitas ou, ainda, que lhes estão sendo negadas oportunidades para alcançar a felicidade e a auto-realização, com independência de seu estado de saúde físico ou das condições sociais e econômicas ${ }^{3}$.

A comunicação, também, é uma das formas que reflete no comportamento do homem, diferenciando-o dos outros animais, pela habilidade de realizar os movimentos faciais e pela produção vocal que lhe possibilita a expressão ${ }^{4}$.

As ações faciais facilitam a aproximação ou o afastamento do outro e, são realizadas devido a uma orientação visual. Expressões de sorriso, surpresa, rancor podem apresentar uma aproximação, uma dúvida ou uma rejeição. Fica claro que 
todos estes gestos, posturas e mímicas estão a serviço da expressão emocional e esta deverá ser a motivação que será associada à resposta motora ${ }^{5}$.

Os movimentos dos músculos faciais, constituintes da chamada expressão ou mímica facial permitem a comunicação não-verbal visando à exteriorização das emoções humanas. Quando há uma alteração desses movimentos faciais, pode ocorrer sequelas funcionais, estéticas, sociais e psicológicas. A limitação desses movimentos faciais é denominada de paralisia facial (PF) que dentre os tipos está a paralisia facial periférica ${ }^{6,7}$.

Além disso, a sociedade nos tempos atuais valoriza, cada vez mais, a estética relacionada à aparência facial, uma vez que a face é o local mais exposto ao meio e os seus traços marcam a individualidade do ser humano ${ }^{8}$.

Diante disso, torna-se viável conhecer a relação da paralisia facial periférica (PFP) adquirida com a QV para corroborar não somente com estudos futuros sobre a temática, mas para alertar os profissionais de saúde e familiares sobre os prejuízos, ou não, que a alteração da mímica facial pode trazer à vida desses indivíduos.

O objetivo deste trabalho foi analisar a qualidade de vida em indivíduos com paralisia facial periférica crônica adquirida.

\section{MÉTODO}

O estudo foi realizado em um Centro de Reabilitação, inserido na Associação dos Deficientes Físicos de Alagoas - ADEFAL. É uma instituição de referência em deficiência física no Estado de Alagoas, que atende a um significante número de pessoas em todo o Estado, com cerca de 1.450 atendimentos diários, em todas as áreas da reabilitação.

Foram incluídos todos os indivíduos com PFP adquirida até 12 meses de acometimento, já que até esse período o indivíduo apresenta muitas expectativas sobre a melhora da PFP e, após esse período pode ter outra percepção acerca da QV, principalmente quando não há melhora dos músculos faciais. Todos foram encaminhados ao Serviço de Fonoaudiologia, durante o período de 2008 a 2009. Não houve restrição quanto ao gênero.

Foram excluídos:

- indivíduos com paralisia facial central, por não ser o objetivo do estudo;

- cadeirantes, pelo fato da dificuldade de deambulação poder se configurar em um impacto possivelmente interferente na $\mathrm{QV}$, o que tornaria este dado um viés neste estudo;

- com alterações faciais congênitas por poder ter se adaptado às mesmas, levando a uma QV diferente dos que tiveram alterações da mímica facial adquiridas após anos de desempenho normal desta função;

- paralisias faciais recidivantes, síndromes maxilo-faciais congênitas e marcas cicatriciais, pelo fato destas alterações poderem influenciar os resultados da avaliação dos músculos da face não correspondendo, portanto, a modificações decorrentes da PFP;

- sujeitos com outras alterações neurológicas e cognitivas relevantes e quadros de afasia, em razão de poderem interferir na compreensão dos comandos verbais e, portanto, na movimentação facial;

- indivíduos que estão em acompanhamento fisioterápico para tratamento da $\mathrm{PF}$, visto que, eles poderão apresentar melhores resultados em todas as avaliações, não correspondendo ao perfil da amostra;

- indivíduos que já realizaram atendimento fonoaudiológico por poderem ter apresentado melhora no quadro da PFP adquirida com diferente percepção da $Q V$ em relação àqueles que nunca realizaram esse atendimento.

Os indivíduos elegíveis para pesquisa foram sujeitos com no mínimo 21 anos, por apresentarem maior probabilidade de ter uma vida social além do convívio familiar, ou seja, um contato com outras pessoas que não somente as mais próximas, e no máximo 70 anos, por acreditar que superior a essa idade, a visão de QV para esses indivíduos possa ser diferente.

Inicialmente, os indivíduos foram identificados mediante a ficha específica realizada sempre de forma individual, contendo os seguintes itens: nome, idade, gênero, profissão, ocupação, escolaridade, data do acometimento, tempo de procura ao serviço de fonoaudiologia, etiologia e se já realizou outros tratamentos, como a fonoaudiologia e a fisioterapia. Aqueles que obedeceram aos critérios foram convidados a participar da pesquisa pelo pesquisador principal, que thes apresentou as informações sobre a pesquisa (objetivos, riscos, benefícios e procedimentos aos quais seriam submetidos), verbalmente e de forma escrita. Após a leitura e esclarecimento das dúvidas do Termo de Consentimento Livre e Esclarecido, o paciente assinava e consentia com a realização e divulgação desta pesquisa e seus resultados conforme Resolução 196/96 (BRASIL. Resolução MS/CNS/CNEP no 196/96 de 10 de outubro de 1996). Esta leitura foi feita pelo próprio sujeito ou pelo pesquisador (na presença de alguma impossibilidade do sujeito ler). Foram assinadas duas vias, ficando uma com o pesquisador, e outra com o voluntário da pesquisa. Foi garantido o direito 
à retirada de consentimento a qualquer momento, deixando de participar do estudo, sem quaisquer tipos de prejuízo ao indivíduo.

Após o consentimento, foi realizada a avaliação da PFP por meio da classificação de House \& Brackmann ${ }^{9}$, referente ao grau de PFP. Essa classificação é constituída de seis graus: I - Normal; II - Disfunção leve; III - Disfunção moderada; IV Disfunção moderadamente severa; V - Disfunção severa; VI - Paralisia total, com descrição de alterações que podem ser observadas em cada grau, abordando a avaliação grosseira da face, em repouso (simetria e tônus) e em movimento fronte, olho e boca.
Posteriormente, foi aplicado um questionário ${ }^{10}$ (Figura 1) por meio de entrevista com perguntas fechadas sobre a opinião do indivíduo com relação à sua face abrangendo os músculos em repouso e em movimento (testa, olho, nariz e lábios), a presença de queixa e grau de incômodo por sincinesias e contraturas e a presença de prejuízo nas atividades sociais e profissionais e grau do mesmo, todos realizados no mesmo dia. Para melhor adequação aos objetivos do estudo as questões um e dois não foram somadas e, sim, analisadas individualmente, assim como também foram realizadas comparações com a classificação do grau da $\mathrm{PF}^{9}$ com as etiologias.

1. Avaliação da face em repouso (graduada de 0 - péssima a 4 - ótima);

2. Avaliação da face ao movimentar a testa, olho, nariz e lábios (graduada de 0 - péssima a 4 - ótima);

Os itens 1 e 2 foram somados e originaram a nota total de auto - avaliação do paciente.

3. Presença de queixa e grau de incômodo por sincinesias e contraturas ( 0 - nenhum a 4 - muito)

4. Presença de prejuízo nas atividades sociais e profissionais e grau do mesmo ( 0 - nenhum a 4 - muito)

\section{Figura 1 - Questionário da avaliação da face}

A variável primária foi a relação da PFP adquirida com a QV, e as variáveis secundárias foram a classificação da PFP por meio de protocolo com a comparação dos resultados obtidos entre a auto-percepção do indivíduo e as etiologias.

Os dados complementares desta pesquisa foram: nome, idade, gênero, profissão, ocupação, escolaridade, data do acometimento, tempo de procura ao serviço de fonoaudiologia, etiologia e se havia realizado algum tratamento, como fisioterápico ou fonoaudiológico, dentre outros.

Foram selecionados 12 indivíduos com PFP adquirida com tempo de acometimento superior a três meses, sendo encontrados, $66,7 \%$ do gênero feminino e 33,3\% do gênero masculino, envolvendo as duas etiologias. Quanto à etiologia foram encontrados $75 \%$ dos indivíduos com PFP de Bell e 25\% com PFP por Schwannoma.

A pesquisa foi aprovada pelos Comitês de Ética em Pesquisa da Universidade Estadual de Ciências da Saúde de Alagoas - UNCISAL e da Universidade Federal de São Paulo - UNIFESP, sob os protocolos 762/07 e 0380/10, respectivamente.

O tipo de estudo foi transversal e os dados coletados foram tabulados e armazenados em uma planilha eletrônica de dados (Microsoft Exce ${ }^{\circledR} 2003$. Redmond, WA, EUA), e analisados pela estatística descritiva, utilizando-se o programa estatístico SPSS, versão 13.0.
Para a comparação da classificação do grau da $\mathrm{PF}^{9}$, a face em repouso e em movimento, a presença de queixa e grau de incômodo por sincinesias e contraturas e a presença de prejuízo nas atividades sociais e profissionais e grau do mesmo foi utilizado o teste exato de Fisher. Para todos os testes foi adotado o nível de significância de 5\%.

\section{RESULTADOS}

A tabela 1 mostra a distribuição demográfica do grupo.

Em relação às sequelas na PFP de Bell, 55,6\% dos indivíduos não apresentaram sequelas e 44,4\% apresentaram. Na PFP por Schwannoma 66,7\% dos indivíduos apresentaram sequelas e 33,3\% não apresentaram.

As tabelas 2 e 3 mostram as relações entre os graus de paralisia e a auto-percepção do indivíduo quanto à face em repouso e em movimento.

A tabela 4 mostra a distribuição da população de acordo com a etiologia, a classificação do grau de paralisia facial e a avaliação da face ao movimentar testa, olho, nariz e lábios.

A tabela 5 mostra a distribuição da população de acordo com a etiologia e a classificação do grau da paralisia facial segundo House \&Brackmann. 
Tabela 1 - Distribuição demográfica do grupo estudado

\begin{tabular}{lcc}
\hline & Bell & Schwannoma \\
\hline Gênero & 3 & 1 \\
feminino & 6 & 2 \\
masculino & 30 & 36 \\
\hline Idade (em anos) & 57 & 45 \\
Mínimo & 39,78 & 40,0 \\
Máximo & & \\
Média & 90 & 130 \\
\hline Tempo de PFP (em dias) até a procura do tratamento & 270 & 240 \\
Mínimo & 138.67 & 173.33 \\
Máximo & & 0 \\
Média & 1 & 1 \\
\hline Escolaridade/Profissão/Ocupação & 2 & 1 \\
Ensino superior completo & 3 & 1 \\
Ensino médio completo & 3 & \\
Ensino fundamental completo & & \\
Ensino fundamental incompleto & & \\
\hline
\end{tabular}

Tabela 2 - Distribuição da população de acordo com a etiologia, classificação do grau da paralisia facial e a auto-percepção do indivíduo da face em repouso

\begin{tabular}{|c|c|c|c|c|}
\hline & \multicolumn{3}{|c|}{$\begin{array}{c}\text { Gradução HB } \\
\text { N (\%) }\end{array}$} & \multirow[t]{2}{*}{ Total } \\
\hline & $|-| I$ & III-IV & V-VI & \\
\hline \multicolumn{5}{|l|}{ Bell } \\
\hline Ótima & $5(100)$ & $1(33,3)$ & 0 & 6 \\
\hline Péssima & 0 & $2(66,6)$ & $1(100)$ & 3 \\
\hline Total & $5(100)$ & $3(100)$ & $1(100)$ & 9 \\
\hline \multicolumn{5}{|l|}{ Schwannoma } \\
\hline Ótima & $2(100)$ & 0 & 0 & $2(66,7)$ \\
\hline Péssima & 0 & 0 & $1(100)$ & $1(33,3)$ \\
\hline Total & $2(100)$ & 0 & $1(100)$ & $3(100)$ \\
\hline
\end{tabular}

*Teste estatístico Mann-Whitney

Tabela 3 - Distribuição da população de acordo com a etiologia, a classificação do grau da paralisia facial e a avaliação da face ao movimentar testa, olho, nariz e lábios

\begin{tabular}{lccccc}
\hline & \multicolumn{3}{c}{$\begin{array}{c}\text { Gradução HB } \\
\text { N (\%) }\end{array}$} & Total \\
\cline { 2 - 4 } & & I-II & III-IV & V-VI & \\
\hline Bell & & & 0 & $4(44,4)$ \\
& Ótima & $4(80)$ & 0 & $1(100)$ & $5(55,6)$ \\
& Péssima & $1(20)$ & $3(100)$ & $1(100)$ & $9(100)$ \\
\hline Total & $5(100)$ & $3(100)$ & 0 & $2(66,7)$ \\
\hline Schwannoma & & & $1(100)$ & $1(33,3)$ \\
& Ótima & $2(100)$ & 0 & $1(100)$ & $3(100)$ \\
\hline Péssima & 0 & 0 & & \\
& Total & $2(100)$ & 0 & &
\end{tabular}

*Teste estatístico Mann-Whitney 
Tabela 4 - Distribuição da população de acordo com a etiologia, a classificação do grau da paralisia facial e a avaliação da face ao movimentar testa, olho, nariz e lábios

\begin{tabular}{|c|c|c|c|c|}
\hline & \multicolumn{3}{|c|}{$\begin{array}{c}\text { Gradução HB } \\
\text { N (\%) }\end{array}$} & \multirow[t]{2}{*}{ Total } \\
\hline & I-II & III-IV & V-VI & \\
\hline \multicolumn{5}{|l|}{ Bell } \\
\hline Ótima & $4(80)$ & 0 & 0 & $4(44,4)$ \\
\hline Péssima & $1(20)$ & $3(100)$ & $1(100)$ & $5(55,6)$ \\
\hline Total & $5(100)$ & $3(100)$ & $1(100)$ & $9(100)$ \\
\hline \multicolumn{5}{|l|}{ Schwannoma } \\
\hline Ótima & $2(100)$ & 0 & 0 & $2(66,7)$ \\
\hline Péssima & 0 & 0 & $1(100)$ & $1(33,3)$ \\
\hline Total & $2(100)$ & 0 & $1(100)$ & $3(100)$ \\
\hline
\end{tabular}

Tabela 5 - Distribuição da população de acordo com a etiologia e a classificação do grau da paralisia facial segundo House \& Brackmann

\begin{tabular}{lcccc}
\hline & \multicolumn{3}{c}{$\begin{array}{c}\text { Gradução HB } \\
\text { N (\%) }\end{array}$} & \multirow{2}{*}{ Total } \\
\cline { 2 - 4 } & I-II & III-IV & V-VI & \\
\hline Bell & $5(55,6)$ & $3(33,3)$ & $1(11,1)$ & $9(100)$ \\
Schwannoma & $2(66,7)$ & $0(0)$ & $1(33,3)$ & $3(100)$ \\
Total & $7(58,3)$ & $3(25)$ & $2(16,7)$ & $12(100)$ \\
\hline
\end{tabular}

*Teste estatístico Mann-Whitney

A tabela 6 mostra a distribuição da população de acordo com a etiologia, a classificação do grau da paralisia facial e percepção de queixa e incômodo por sincinesias e contraturas.
A tabela 7 mostra a distribuição da população de acordo com a etiologia, a classificação do grau da paralisia facial e a presença de prejuízo nas atividades sociais e profissionais e grau do mesmo.

Tabela 6 - Distribuição da população de acordo com a etiologia, a classificação o grau da paralisia facial e percepção de queixa e incômodo por sincinesias e contraturas

\begin{tabular}{|c|c|c|c|c|}
\hline & \multicolumn{3}{|c|}{$\begin{array}{c}\text { Gradução HB } \\
\text { N }\end{array}$} & \multirow[t]{2}{*}{ Total } \\
\hline & I-II & IIIIIV & V-VI & \\
\hline \multicolumn{5}{|l|}{ Bell } \\
\hline Nenhum & 5 & 0 & 0 & 5 \\
\hline Muito & 0 & 3 & 1 & 4 \\
\hline \multicolumn{5}{|l|}{ Schwannoma } \\
\hline Nenhum & 2 & 0 & 0 & 2 \\
\hline Muito & 0 & 0 & 1 & 1 \\
\hline
\end{tabular}


Tabela 7 - Distribuição da população de acordo com a etiologia, a classificação do grau da paralisia facial e a presença de prejuízo nas atividades sociais e profissionais e grau do mesmo

\begin{tabular}{|c|c|c|c|c|}
\hline & \multicolumn{3}{|c|}{$\begin{array}{c}\text { Gradução HB } \\
\mathbf{N}\end{array}$} & \multirow[t]{2}{*}{ Total } \\
\hline & I-II & III-IV & V-VI & \\
\hline \multicolumn{5}{|l|}{ Bell } \\
\hline Nenhum & 5 & 0 & 0 & 5 \\
\hline Muito & 0 & 3 & 1 & 4 \\
\hline \multicolumn{5}{|l|}{ Schwannoma } \\
\hline Nenhum & 2 & 0 & 0 & 2 \\
\hline Muito & 0 & 0 & 1 & 1 \\
\hline
\end{tabular}

\section{DISCUSSÃO}

A PF altera movimentos faciais que podem resultar em desfiguração do rosto em repouso, bem como em movimento ou nas expressões espontâneas. Dificuldades particulares envolvem respostas reativas em comunicação social e psicológica e de fadiga física relacionada às interações sociais ${ }^{24}$.

Observamos na literatura que os instrumentos de avaliação de QV geralmente são construídos em países de língua inglesa, para posteriormente, serem traduzidos e aplicados em diferentes culturas. Os instrumentos específicos avaliam de forma individual e específica determinados aspectos da QV, são mais sensíveis para detectar alterações após uma intervenção. Já os instrumentos genéricos foram desenvolvidos para demonstrar o impacto de uma doença sobre a vida de pacientes em uma grande variedade da população ${ }^{17}$. Entre os genéricos há World Health Organization Quality Life (WHOQL) e o The Medical Outcomens Study 36-item Short-Form Health Survey (SF-36) traduzidos para o português na versão brasileira, e muito utilizado na avaliação de QV de diferentes grupos de pessoas ${ }^{18}$. O conceito de QV, assim como seus instrumentos de avaliação, ainda está em processo de desenvolvimento ${ }^{19}$.

Os questionários elaborados para uma cultura específica podem não apresentar os mesmos objetivos e significados para a cultura de outro país. O conceito de "boa qualidade", por ser abstrato e ambíguo, admite múltiplos significados, para diferentes pessoas, locais ou ocasiões. Essa multiplicidade de significados, colocados de forma tão heterogênea, dificulta comparações ${ }^{15,16}$.

A utilização desses questionários em culturas diversas recebe muitas críticas em decorrência das peculiaridades de determinadas populações ${ }^{20}$. Não foram utilizados nesse estudo questionários validados para a QV por serem genéricos, podendo comprometer a pesquisa, optando-se por questionários com perguntas fechadas e já utilizadas em estudo anterior por profissionais específicos da área.

A classificação do grau da $\mathrm{PF}^{9}$ foi também utilizada por outras pesquisas $8,21,22$. A necessidade de se estabelecer prognóstico quanto à evolução da PFP, tem levado ao desenvolvimento de métodos para quantificá-la clinicamente. Diversos métodos subjetivos e objetivos têm sido propostos para essa avaliação. Os métodos baseiam-se na presença ou na ausência de certos movimentos faciais pré-estabelecidos. Dentre eles, destacam-se a graduação de House e Brackmann ${ }^{9}$, de Yanagihara e o Facial Grading System ${ }^{23}$, além do instrumento Facial Disability Index utilizado na avaliação de pacientes com transtornos do nervo facial24.

A avaliação clínica do grau de PFP varia de acordo com o examinador, sendo que dentre os sugeridos, a classificação de House \& Brackmann ${ }^{9}$ para a quantificação da PF é o mais utilizado na literatura internacional, avaliando a musculatura orofacial em repouso e durante os movimentos, visando comparar a hemiface paralisada e a outra sem alteração ${ }^{25}$. Todas as escalas de graduação para PFP possuem suas imperfeições. Vários centros de reabilitação do nervo facial empregam métodos de avaliação da PFP, tais como os critérios de classificação de House \& Brackmann ${ }^{9}$ e o Sistema de Graduação Facial de Ross, Fradet e Nedzelski².

Quanto ao gênero e a idade, Latorre et al. ${ }^{27}$ referiram que ambos não interferiram para o desenvolvimento de uma PFP. Pacientes mais jovens - especialmente abaixo de 20 anos, apresentaram prognóstico melhor em relação a pacientes mais idosos - acima de 61 anos $^{28}$. Homens e mulheres são igualmente afetados, embora a incidência seja maior em mulheres grávidas ${ }^{11}$.

Freitas et al. ${ }^{10}$ encontraram que em alguns casos classificados como grau leve pela avaliação fonoaudiológica, foram referidos sintomas de muito 
incômodo pelo paciente, e graus severos foram relatados com pouco incômodo pelo paciente. Com isso, os autores acreditam que além da questão orgânica, há outros fatores que levam a um maior ou menor incômodo, como fatores psicossociais. Na presente pesquisa, pela classificação de House \& Brackmann ${ }^{9}$ foram encontrados na PFP de Bell $55,6 \%$ dos indivíduos com PFP do grupo de graus I-II (normal e disfunção leve), 33,3\% do grupo de graus III-IV (Disfunção moderada e disfunção moderadamente severa) e $11,1 \%$ do grupo de graus V-VI (Disfunção severa e paralisia total). Na PFP por Schwannoma $66,7 \%$ no grupo de graus I-II (normal e disfunção leve) e 33,3\% no grupo de graus V-VI (Disfunção severa e paralisia total).

Aproximadamente um terço dos pacientes evoluiu com sequelas estéticas ou funcionais significantes como: disfunção oral, contraturas, obstrução nasal, sincinesias e espasmo hemifacial. A PFP idiopática pode deixar sequelas cosméticas e funcionais importantes, bem como a recidiva da paralisia em parcela significante dos pacientes. A recuperação completa somente é possível nos casos de lesão transitória do nervo (neuropraxia). Quando a lesão é mais grave, a recuperação é acompanhada de sequelas ${ }^{29}$. Nesse estudo, na PFP de Bell e por Schwannoma a maioria não referiu queixa e grau de incômodo por sincinesias e contraturas para o grupo de graus I-II.

No estudo de Coulson et al. ${ }^{2}$ foram identificados aspectos da expressão emocional afetados pela PF com uma redução significante na relação social. As causas que a geram são múltiplas, produzindo uma deformidade estética e funcional, bem como distúrbios emocionais, sociais e profissionais ${ }^{30}$. Nesse estudo, foi perguntado aos entrevistados se houve prejuízo nas atividades sociais e profissionais, sendo encontrado na PFP de Bell 55,6\% indivíduos que não relataram prejuízo para o grupo de graus I-II e $44,4 \%$ com relato de muito prejuízo. Na PFP por Schwannoma $66,7 \%$ dos indivíduos relataram nenhum prejuízo também para o grupo de graus I-II e 33,3\% muito prejuízo. Não houve respostas intermediárias, podendo ser atribuído a fatores psicossociais, em que utilizaram a ausência de queixas e o sentir incômodo e o prejuízo, como aspectos extremos.
A maioria dos pacientes tinha condição funcional praticamente normal (grau II), por isso apresentaram poucas queixas. Por outro lado, todos os 5 pacientes com graus a partir de III tiveram queixas maiores e maior incômodo.

No presente estudo foram entrevistados 35 indivíduos, sendo encontradas em 22 indivíduos as duas etiologias mais frequentes (PFP de Bell e por Schwannoma). Sendo considerados os indivíduos com PFP adquirida com tempo de acometimento superior a três meses, totalizando 12 indivíduos, sendo essa amostra descrita no trabalho.

Em cerca de $70 \%$ dos pacientes com PF de Bell, a função motora voluntária normaliza espontaneamente, principalmente dentro três meses; os outros $30 \%$ permanecem com uma paralisia, com sequelas que variam de muito suave a muito grave $^{13}$. No estudo de Bauso ${ }^{11}$ há o relato que entre 80 e $90 \%$ se recuperam satisfatoriamente.

$\mathrm{Na}$ área da fonoaudiologia vários trabalhos abordam a terapia fonoaudiológica como recurso para tratar os pacientes acometidos pela PFP. Esse trabalho já é consagrado, porém ainda encontram-se dificuldades em obter um método de avaliação que seja prático, objetivo, de baixo custo e que possa ser usado por fonoaudiólogos no acompanhamento e na determinação da alta ${ }^{26}$.

Na PFP adquirida o indivíduo se vê desfigurado e não se reconhece ao espelho. Essa manifestação gera angústia, dificuldade de relacionamento social e profissional. Os dados encontrados nesta pesquisa podem ser utilizados em estudos futuros para analisar a QV em indivíduos acometidos pela PFP adquirida, podendo também ser analisados antes e após o tratamento fonoaudiológico.

\section{CONCLUSÃO}

A paralisia facial periférica crônica adquirida interferiu na qualidade de vida dos indivíduos com graus considerados mais graves.

Tornam-se necessárias outras pesquisas que contemplem a qualidade de vida e a paralisia facial periférica crônica adquirida, uma vez que não foram encontrados estudos suficientes que envolvessem as duas temáticas, principalmente no Brasil. 


\section{ABSTRACT}

Purpose: to analyze quality of life in subjects with acquired chronic peripheral facial palsy. Method: 12 subjects with acquired facial palsy were selected through screening during the sequel, with etiologies of Bell and acoustic schwannoma after resection. The degree of acquired facial palsy was verified, as well as a closed questions interview about complaints with the facial movement was carried out, in order to check if there was interference from facial palsy in the social life of each subject. This was a cross-sectional study. Non-parametric Mann-Whitney and Fisher's exact test, with significance level of $5 \%$, were used in order to analyze the data. Results: the degree of facial palsy were divided as it follows: I-II (Normal to mild dysfunction), III-IV (moderate to moderately severe dysfunction) and V-VI (complete palsy severe dysfunction), according to House \& Brackmann. In the answers about difficulties in professional and personal activities, Bell's palsy individuals with normal to mild dysfunction have no complaints, in moderate to moderately severe dysfunction all answered very severe complaints and in an individual with complete palsy reported a lot of complaints. In the acoustic Schwannoma individuals, in the group classified as mild dysfunction, all answered no damage complaints, while among those with severe to complete palsy, one individual reported a lot of complaints in professional and personal activities. Conclusion: the acquired chronic peripheral facial palsy interfered with quality of life in subjects with more severe degrees of palsy.

KEYWORDS: Quality of Life; Facial Palsy; Bell Palsy; Facial Expression; Psychosocial Impact

\section{REFERÊNCIAS}

1. Brach JS, VanSwearingen JM, Lenert J, Johnson PC. Facial neuromuscular retraining for oral synkinesis. Plast Reconstr Surg. 1997;99(7):192231; discussion 1932-3. Comment in: Plast Reconstr Surg. 1998;101(2):554-5. Plast Reconstr Surg. 2003;111(7):2370-5.

2. Coulson SE, O'dwyer NJ, Adams RD, Croxson GR. Expression of emotion and quality of life after facial nerve paralysis. Otol Neurotol. 2004;25(6):1014-9.

3. Organización Mundial de la Salud. Promoción de la salud. Glosario. Genebra: OMS; 1998. p. 1-35.

4. Lazarini PR, Fernandes AMF. Anatomia do nervo facial. In: Lazarini PR, Fouquet ML. Paralisia facial: Avaliação, tratamento e reabilitação. São Paulo: Lovise; 2006. p. 1-10.

5. Guedes ZCF. Reabilitação fonoaudiológica nas paralisias faciais congênitas. In: Lazarini PR, Fouquet ML. Paralisia facial: Avaliação, tratamento e reabilitação. São Paulo: Lovise; 2006. p. 169-72.

6. Lazarini PR, Fernandes AMF, Brasileiro VSB, Custódio SEV. Paralisia facial periférica por comprometimento do tronco cerebral - A propósito de um caso clínico. Rev Bras Otorrinolaringol. 2002 Mai; 68 (1): 140-4.

7. Calais LL, Gomez MVSG, Bento RF, Comerlatti LR. Avaliação funcional da mímica na paralisia facial central por acidente cerebrovascular. Pró-Fono R. Atual. Cient. 2005 Mai/ Ago; 17 (2): 213-22.
8. Veronezi RJB. Análise tardia do grau de paralisia facial em pacientes operados de schwannoma vestibular [dissertaᄀção]. São Paulo: Universidade Estadual de Campinas. Faculdade de Ciências Médicas; 2006.

9. House JW, Brackmann DE. Facial nerve grading system. Otolaryngol Head Neck Surg. 1985; 93(2):146-7.

10. Freitas KCS, Goffi Gómez MV. Grau de percepção e incômodo quanto à condição facial em indivíduos com paralisia facial periférica na fase de sequelas. Rev Soc Bras Fonoaudiol. 2008 Jun; 13 (2): 113-8.

11. Bauso D. Parálisis facial idiopática o parálisis de Bell. Evid Act Pract Ambul. 2006 Febr; 9(1): 22-5.

12. Tessitore A, Pfeilsticker LN, Paschoal JR. Aspectos neurofisiológicos da musculatura facial visando a reabilitação na paralisia facial. Rev Cefac. 2008 Jan/ Mar; 10 (1): 68-75.

13. Beursken $\mathrm{CH}$, Heymans PG. Physiotherapy in patients with facial nerve paresis: Description of outcomes. American Journal of Otolaryngology. 2004. Nov/ Dec; 25 (6): 394-400.

14. Garanhani MR, Cardos JR, Capelli AMG, Ribeiro MC. Fisioterapia na paralisia facial periférica: estudo retrospectivo. Rev Bras Otorrinolaringol. 2007 Jan/ Fev; 73 (1): 112-5.

15. Niv D, Kreitler S. Pain and quality of life. Pain. 2001; 1(3): 150-61.

16. Skevington S. Advancing cross-cultural research on quality of life: Observations drawn from the WHOQOL development. 2002; 11: 135-44. 
17. Duarte PS, Miyazaki MCOS, Ciconelli RM, Sesso R. Tradução e adaptação cultural do instrumento de avaliação de qualidade de vida para pacientes renais crônicos (KDQOL-SF TM). Rev Assoc Med Bras. 2003;49(4):375-81.

18. Cruz KCT. Avaliação da capacidade funcional e da qualidade de vida em indivíduos com acidente vascular encefálico com igual maior ou igual a 55 anos [dissertaᄀção].São Paulo: Universidade Estadual de Campinas. Faculdade de Ciências Médicas; 2004.

19. Castro M, Caiuby AVS, Draibe AS, Canziani MEF. Qualidade de vida de pacientes com insuficiência renal crônica em hemodiálise avaliada através do instrumento genérico SF-36. Rev Assoc Med Bras. 2003 Jul/ Set; 49 (3): 245-9.

20. Aguiar CCT, Vieira APGF, Carvalho AF, Montenegro RMJ. Instrumentos de avaliação de qualidade de vida relacionada à saúde no diabetes melito. Arq Bras Endocrinol Metab. 2008 Ago 52 (6): 931-9.

21. Silva ECCF, Testa JRG, Fukuda Y. Achados fonoaudiológicos em pacientes submetidos a anastomose hipoglosso facial. Rev Bras Otorrinolaringol. 2003 Mai/ Jun; 69 (3): 377-84.

22. Kanzaki J, Tos M, Sanna M, Moffat DA. New and modified reporting systems from the consensus meeting on systems for reporting results in vestibular schwannoma. Otol Neurotol. 2003;24:642-8.
23. Quintal M, Tessitore A, Paschoal Jr, Pfeilsticker IN. Quantificação da paralisia facial com paquímetro digital. Rev CEFAC. 2004 Abr/ Jun; 6 (2): 170-6.

24. VanSwearingen JM, Brach JS. The facial disability index: reliability and validity of a disability assessment instrument for disorders of the facial neuromuscular system Physical Therapy. 1996 Dec; 76 (12):1288-313.

25. Bento RF, Neto RVB. Tratamento das paralisias faciais - presente e futuro. In: Lavinski L (Org.). Tratamento em Otologia. Rio de Janeiro: Revinter, 2005. p. 709-15.

26. Bernardes DFF, Goffi Gomez MVS, Bento RF. Eletromiografia de superfície em pacientes portadores de paralisia facial periférica. Rev Cefac. 2010 Jan/Fev; 12 (1): 91-6.

27. Latorre EIP, Pérez OGR, Undargaraím LO, Pasin NA. Parálisis facial periférica a frígori. Terapia acupuntural. Archivo Médico De Camaguey. 2004; 8(2): 25-32.

28. Kasse CA, Cruz OLM, Leonhardt FD, Testa JRG, Ferri R, Viertler EY. Valor prognóstico de dados clínicos em paralisia de Bell. Rev Bras Otorrinolaringol. $2005 \mathrm{Jul} / \mathrm{Ago} ; 71$ (4): 454-8.

29. Valença MM, Valença LPA, Lima MCM. Paralisia facial periférica idiopática de Bell: a propósito de 180 pacientes. Arq Neuro-Psiquiatr. 2001 set; 59 (3): 778-83.

30. Rondon H. Parálisis Facial Periférica. Rev Med Clin Condes. 2009; 20(4): 528-35.
http://dx.doi.org/10.1590/S1516-18462012005000023

RECEBIDO EM: 24/05/2011

ACEITO EM: 17/09/2011

Endereço para correspondência:

Rayné Moreira Melo Santos

Rua Engenheiro Mário de Gusmão, 674/902

Ponta Verde - Maceió - AL

CEP: 57035-000

E-mail: raynefono@yahoo.com.br 